\title{
Conformity and statistical tolerancing
}

\author{
Laurent Leblond ${ }^{1}$ and Maurice Pillet, ${ }^{2, *}$ \\ ${ }^{1}$ Groupe PSA, Centre Technique de Vélizy A, 78943 Vélizy-Villacoublay, France \\ ${ }^{2}$ Université Savoie Mont Blanc, Laboratoire SYMME \& IUT Annecy, Département QLIO, Chambéry, France
}

Received: 3 July 2017 / Accepted: 24 October 2017

\begin{abstract}
Statistical tolerancing was first proposed by Shewhart (Economic Control of Quality of Manufactured Product, (1931) reprinted 1980 by ASQC), in spite of this long history, its use remains moderate. One of the probable reasons for this low utilization is undoubtedly the difficulty for designers to anticipate the risks of this approach. The arithmetic tolerance (worst case) allows a simple interpretation: conformity is defined by the presence of the characteristic in an interval. Statistical tolerancing is more complex in its definition. An interval is not sufficient to define the conformance. To justify the statistical tolerancing formula used by designers, a tolerance interval should be interpreted as the interval where most of the parts produced should probably be located. This tolerance is justified by considering a conformity criterion of the parts guaranteeing low offsets on the latter characteristics. Unlike traditional arithmetic tolerancing, statistical tolerancing requires a sustained exchange of information between design and manufacture to be used safely. This paper proposes a formal definition of the conformity, which we apply successively to the quadratic and arithmetic tolerancing. We introduce a concept of concavity, which helps us to demonstrate the link between tolerancing approach and conformity. We use this concept to demonstrate the various acceptable propositions of statistical tolerancing (in the space decentring, dispersion).
\end{abstract}

Keywords: conformity principle / statistical tolerancing / robust engineering

\section{Introduction}

Designing and manufacturing an industrial product are the result of an approach which has the following objective: ensure the functional product requirements for customer satisfaction at the lowest possible cost, as soon as possible and at the expected quality levels. Nevertheless, the parts manufacturing constituting a product can never be produced to target, irrespective of the machine precision used.

Tolerancing limits this variability. It is carried out based on construction hypotheses, which will have to be verified by the production. It represents the verification of the conformity. This conformity is often interpreted as individual tolerancing conformity for each characteristic.

In this paper, we propose a formal definition of statistical conformity that does not apply individually to a part but to a part population. We apply this definition to different acceptance definition: triangular, rectangular and inertial. Each of these definitions satisfies a concavity criterion that we define. We show in appendix the interest of this particular criterion in the case of a statistical tolerancing.

\footnotetext{
* Corresponding author: maurice.pillet@univ-smb.fr
}

\section{Issues, ratings and assumptions}

The variability of parts is taken into account by specifying the limits of the specifications of each elementary characteristic for quantitative and measurable quantitative quantities [1]. The tolerance interval (TI) is the difference between the maximum and minimum permissible values, and therefore shows the permissible variation of a quantity.

The TIs specification for interrelated characteristics is established according to two possibly complementary strategies: the analysis and the synthesis of tolerances [2]. The analysis consists in verifying that the TIs, defined by the functional characteristics required on the product, are respected by the tolerances on the elementary characteristics. The synthesis of the tolerances consists in allocating the tolerances to the basic characteristics from those on the functional requirements.

Formally, a characteristic is a quadruplet $(Y, v, c, R)$ where $Y$, which denotes a quantity, is a real random variable with a probability density, ${ }^{1} \quad v \in \mathbb{R}$ is called nominal, $c \in \mathbb{R}$ is center and $R \in \mathbb{R}_{+}^{*}$ is the radius of a

\footnotetext{
${ }^{1}$ All the real random variables considered in this article are in the same probability space and will always be integrable squares (i.e. admitting a variance).
} 


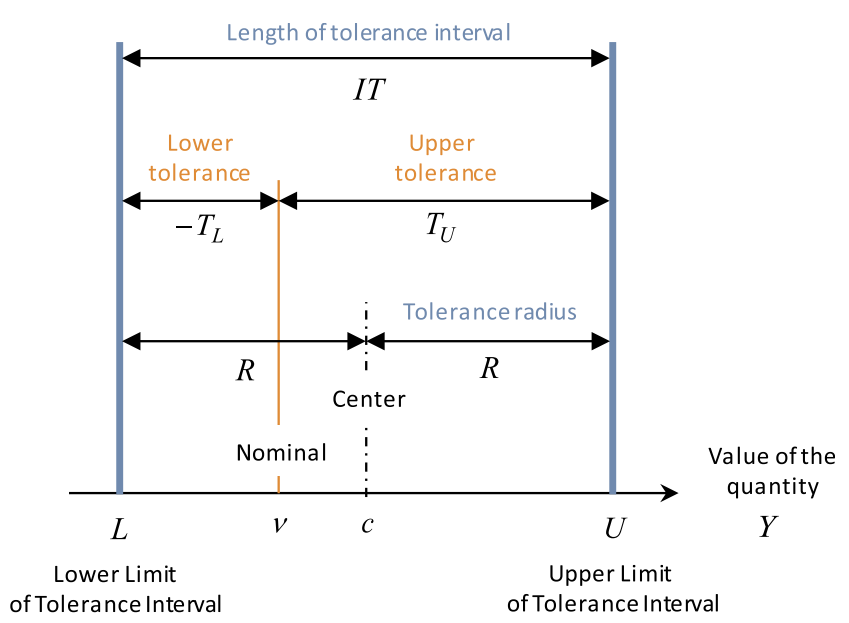

Fig. 1. Illustration of quantities associated with a characteristic.

so-called TI. If the context permits, the quantity $Y$ and the characteristic $(Y, v, c, R)$ will be associated and described using the following quantities (see Fig. 1):

- A TI $[L, U]$ and its length TI:

$U=c+R, L=c-R$ and $T I=U-L=2 R$.

- An upper tolerance (respectively lower): $T_{U}=U-v$ (respectively $T_{L}=L-v$,).

A characteristic is coherent if $v \in[L, U]$ and in that case $T_{U}>0$ and $T_{L}<0$. It is called the nominal centered if $v=c$. In these conditions, $T_{U}=-T_{L}$ and $v=\frac{U+L}{2}$ is at the center of $[L, U] .^{2}$

For a characteristic $(Y, v, c, R), \mu_{Y}=E(Y)$ is the expectation of $Y$ and $\sigma_{Y}=\sqrt{V(Y)}$ its standard deviation. The decentering of this characteristic is $\delta_{Y}=\mu_{Y}-c$. It is centered if $\mu_{Y}=c$ (i.e. $\delta_{Y}=0$ ). When the characteristic is indexed and does not cause confusion, only the index will appear in the denomination of expectation, standard deviation or decentering.

In general, a functional requirement is a function of elementary characteristics. The tolerancing consists in modelling the behaviour in the target vicinity of each of the elementary characteristics. Consequently, the Taylor expansion near the target makes it possible to obtain a linear relationship that relates the functional requirement to the elementary characteristics.

In these conditions, a functional requirement, denoted $Y_{\text {Clearance }}$, is expressed as a linear function of $n$ independent characteristics $\left(Y_{i}\right)_{i=1 \ldots n}$. There are then reals $\left(a_{i}\right)_{i=1 \ldots n}{ }^{3}$ such as:

\footnotetext{
$\overline{2}$ The notion of centered nominal characteristic is totally different from the notion of centered characteristic which means that a process makes it possible to produce the characteristic, on average, at the center of its tolerance interval. In other words, it is not because the nominal of a characteristic is at the center of its tolerance interval that this characteristic is centered. Unfortunately, the usual vocabulary makes confusion frequent and leads to misinterpretations.

${ }^{3}$ These real coefficients of influence are, in general, for a chain of dimensions equal to \pm 1 .
}

$$
\begin{gathered}
Y_{\text {Clearance }}=\sum_{i=1}^{n} a_{i} Y_{i} . \\
\mu_{\text {Clearance }}=\sum_{i=1}^{n} a_{i} \mu_{i} \text { and } \sigma_{\text {Clearance }}^{2}=\sum_{i=1}^{n} a_{i}^{2} \sigma_{i}^{2} .
\end{gathered}
$$

We will also make the following assumptions ${ }^{4}$ :

$$
\nu_{\text {Clearance }}=\sum_{i=1}^{n} a_{i} v_{i} \quad \text { and } \quad c_{\text {Clearance }}=\sum_{i=1}^{n} a_{i} c_{i} .
$$

The tolerancing question is asked like this: Which relationship between $R_{\text {Clearance }}$ and the n-uplet $\left(R_{i}\right)_{i=1 \ldots n}$ makes it possible to verify the following principle of conformity: "If all the characteristics $\left(Y_{i}\right)_{i=1 \ldots n}$ are conformed, then $Y_{\text {Clearance }}$ is conformed, too"?

The most natural notion of conformity in the worst case is expressed as follows: if for all $i=1 \ldots n$, and all the $Y_{i}$ characteristics are within the TI $\left[L_{i}, U_{i}\right]$ then the $Y_{\text {Clearance }}$ fulfilments have to be within the TI [ $\left.L_{\text {Clearance }}, U_{\text {Clearance }}\right]$. This formulation of conformity justifies the following formulae:

- Arithmetic tolerance:

$$
R_{\text {Clearance }}=\sum_{i=1}^{n}\left|a_{i}\right| R_{i} .
$$

In other words, the designer who uses this tolerance to verify that $R_{\text {Clearance }}$ guarantees the assembly functionality from knowledge of the $R_{i}$ does not wish to take any risk: if the production is conform to the tolerances of the basic characteristics, it is certain that the functional requirement will be within the tolerance.

This tolerancing is often too restrictive because it is based on the condition that all the elementary characteristics are at the limits of specification. In order to be more flexible, a second formulae is available to the designer:

- Statistical tolerance (or augmented quadratic) ${ }^{5}$ :

$$
R_{\text {Clearance }}^{2}=\left(\frac{6}{h}\right)^{2} \sum_{i=1}^{n} a_{i}^{2} R_{i}^{2} .
$$

For the tolerance radii $R_{i}$ of the given characteristic, the tolerance radius $R_{\text {Clearance }}$ obtained by this formula is smaller than that obtained by using the arithmetic tolerancing formula, which can greatly facilitate the life of the designer who has a functional constraint on $R_{\text {Clearance. }}$

This formula is traditionally justified in the following way: if all the characteristics $Y_{i}$ follow a normal average law $\mu_{i}=c_{i}$ and standard deviation $\sigma_{i}$, then $Y_{\text {Clearance }}$ follows a

\footnotetext{
${ }^{4}$ These relationships cannot be justified by identifying mean and nominal or centers and using the relation on the averages (2). It should be noted that if the characteristics are, on average, at nominal or at the center, there is no evidence that the resultant characteristic is on average at nominal or center.

${ }^{5}$ Factor $h>0$ is a weighting factor introduced to ease certain constraints of the quadratic tolerancing [13]. If $h=6$, it is the quadratic tolerancing strictly speaking and when $h=2 \sqrt{3}$, it is called probabilistic tolerancing.
} 
normal mean law $\mu_{\text {Clearance }}=c_{\text {Clearance }}$ and standard deviation $\sigma_{\text {Clearance }}$ according to (2). The statistical tolerancing formulae are deduced by assuming that tolerance rays are proportional to the standard deviations: $R_{i}=\frac{6}{h} 3 \sigma_{i}$ and $R_{\text {Clearance }}=3 \sigma_{\text {Clearance. }}$. In these conditions, $\mathbb{P}\left(Y_{\text {Clearance }} \notin[\right.$ $\left.\left.L_{\text {Clearance }}, U_{\text {Clearance }}\right]\right) \leq 0,135 \%$ and the risk of having a noncompliant clearance seems to be under control.

However, this justification is not satisfactory since it uses manufacturing parameters which are unknown to the designer under the restrictive and unrealistic nullity hypothesis of the decentring of the characteristics.

The aim of this article is to show that a correct justification of the statistical tolerancing formulae requires defining specific conformity criteria. In order to verify the conformity, the manufacturer must take the tolerancing method of the characteristics produced into account. Sometimes "to be within tolerances" is not sufficient. Designers should not ignore this fact when they use statistical tolerancing. Otherwise they will justify a design with high risks. The management of information between design and manufacturing is thus affected.

\section{Proposition of a definition of conformity for statistical tolerancing}

The acceptance of the conformity by the sole respect of the TI is too restrictive. Indeed, it is not the measurements of characteristics carried out on a limited set of production that are to be considered, but rather the conformity of what the process can potentially produce, namely, the population of parts. In other words, it is not so much an effective part that one has to judge ${ }^{6}$ but the capacity to produce parts in a sufficiently homogeneous way to guarantee their assembly: one must therefore judge the conformity of the process that produces them.

Definition 1-Conformity.

A conformity $C=\left(k,\left(r, \xi_{r}^{o b j}\right)\right)$ is the data of a context $k \in \mathbb{R}_{+}^{*}$ and a couple $\left(r, \xi_{r}^{o b j}\right)$ called conformity objective where $r:[0,1] \rightarrow[0,1]$, is decreasing and strictly positive on $\left[0,1\left[, r(1)=0, \xi_{r}^{o b j} \in \mathbb{R}_{+}^{*}\right.\right.$ and $\sup _{u \in[0,1]}\left(r(u)+\xi_{r}^{o b j} u\right) \leq 1$.

The application $r$ is a type of objective and a goal is maximal if the preceding inequality is equality.

A characteristic at the centered nominal $(Y, v, R)$ is called $C$-conformed ${ }^{7}$ if:

$$
\begin{gathered}
\frac{\left|\delta_{Y}\right|}{R}<\xi_{r}^{o b j} \quad \text { and } \frac{\sigma_{Y}}{R} \leq \frac{1}{k} r\left(\frac{\left|\delta_{Y}\right|}{\xi_{r}^{o b j} R}\right) . \\
\delta_{Y}=\mu_{Y}-c
\end{gathered}
$$

Affirming that a characteristic conformed according to this definition, is to say that, up to a certain limit, the more decentering, the more the variability must be small in

\footnotetext{
${ }^{6}$ A given part is actually conformed if these characteristics meet the tolerance intervals. One-part conformance is therefore a binary problem modulo the measurement process.

7 The definitions given here in the case of one-dimensional characteristics for the sake of simplification can be generalized to the multidimensional case.
}

proportion to the tolerance radius. The way in which the reduction of this variability is managed through the type of objective ( $r$ application) qualifies the nature of the conformity considered. We shall see that the choice of a concave as a type of objective ${ }^{8}$ conditions the expected properties of a quadratic tolerancing.

We considered the following three types of objectives with $\left.\left(c_{P}, c_{I}\right) \in\right] 0,1[2$ and $\mathrm{u} \in[0,1]$ :

Objective of the tolerance or triangular:

$$
r_{T}(u)=1-u \text { and } 0<\xi_{r_{T}}^{o b j} \leq 1=\xi_{r_{T}}^{\max } .
$$

\section{Performance objective or rectangular:}

$$
r_{P}(\mathrm{u})=c_{P} \mathrm{I}(\mathrm{u}) \text { and } 0<\xi_{r_{P}}^{o b j} \leq 1-c_{P}=\xi_{r_{P}}^{\max } .
$$

\section{Extended or elliptical inertial objective:}

$$
r_{I}(u)=c_{I} \sqrt{1-u^{2}} \text { and } 0<\xi_{r_{P}}^{o b j} \leq \sqrt{1-c_{I}^{2}}=\xi_{r_{P}}^{\max } .
$$

For a conformity $C=\left(k,\left(r, \xi_{r}^{o b j}\right)\right)$, these denominations come from the surface form represented in the plane $\%$ decentering $\times \%$ standard deviation (Fig. 2) where the percentage is relative to a radius of tolerance [3]: Surface $_{k}\left(r, \xi_{r}^{o b j}\right)=\left\{(x, y) \in-\xi_{r}^{o b j}, \xi_{r}^{o b j} \times \mathbb{R}_{+}^{*} / y \leq \frac{1}{k} r\left(\frac{|x|}{\xi^{o b j}}\right)\right\}$.

A characteristic $\mathrm{Y}$ is conformed according $\xi_{\mathrm{to}}$ a conformity defined by a particular surface if the couple $\left(\frac{\delta}{R}, \frac{\sigma}{R}\right)$ is a point inside this surface.

We demonstrate in the appendix that the surface defining compliance must:

- on the one hand is included in the triangle so the base is equal to the arithmetic tolerancing and the height is equal to the dispersion of the quadratic tolerancing;

- on the other hand be a concave surface. Rectangle, circle and ellipse are concave surface.

In the example (Fig. 2) below, the characteristic is extended inertial and tolerance-compliant but is not performance-compliant. Rectangle and Ellipse are inside the triangle but does not cover the available area.

This definition of conformity covers the most frequent definitions, in particular those using capability indicators [4]. Indeed, each of the preceding conformity criteria can be expressed using the traditional capability indicators $[5,6]^{9}$ associated with a characteristic $(Y, v, R)$ :

\footnotetext{
${ }^{8}$ Recall us that $r:[0,1] \rightarrow[0,1]$ is concave if for any $\lambda \in[0,1]$ and $(x, y) \in[0,1]^{2}, r(\lambda x+(1-\lambda) y) \geq \lambda r(x)+(1-\lambda) r(y)$.

${ }^{9}$ These indicators are defined without any reference to the form of the law of $Y_{l}$ even if the coefficient 3 comes from considerations of the normal law. The capability indexes which are defined by using their denominator as the length of a fluctuation interval at $99.73 \%$, for example, are not included here because they are too dependent on the law of $Y_{l}$, making it difficult to exploit. Usually, $P p$ is said to be the potential coefficient of capability, $P p k$ the coefficient of performance and Ppm the capability coefficient of Taguchi.
} 


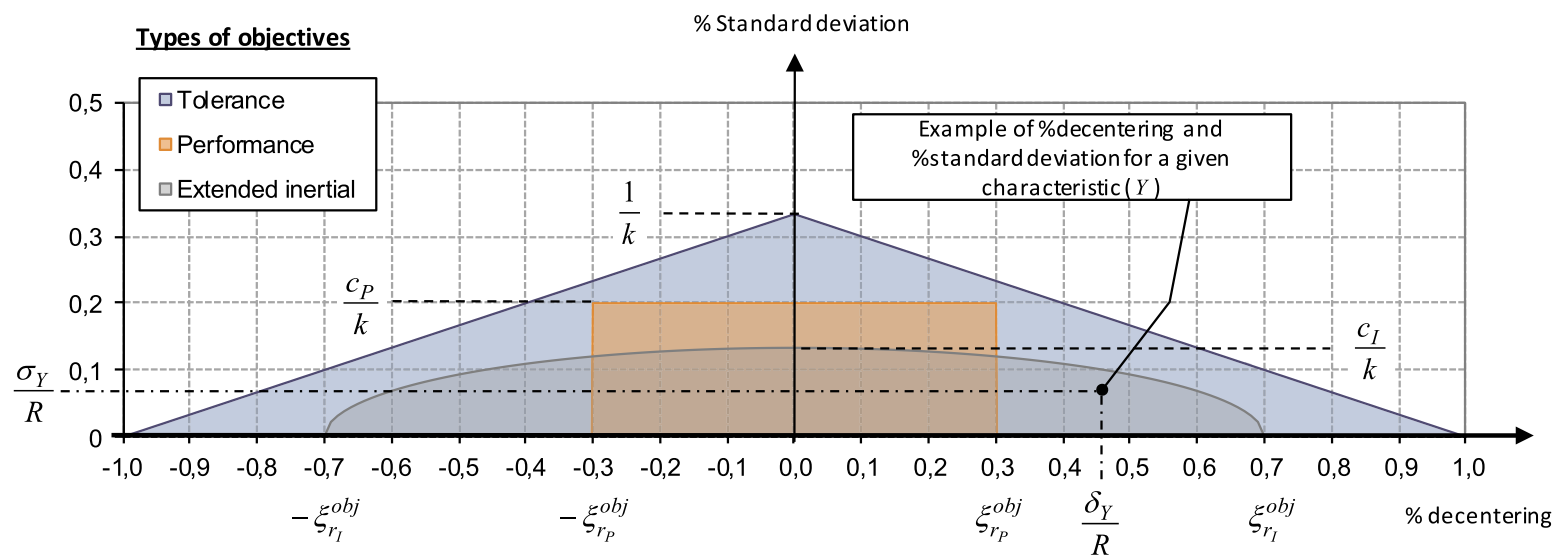

Fig. 2. Illustration of surfaces defined by conformities for $k=3$.

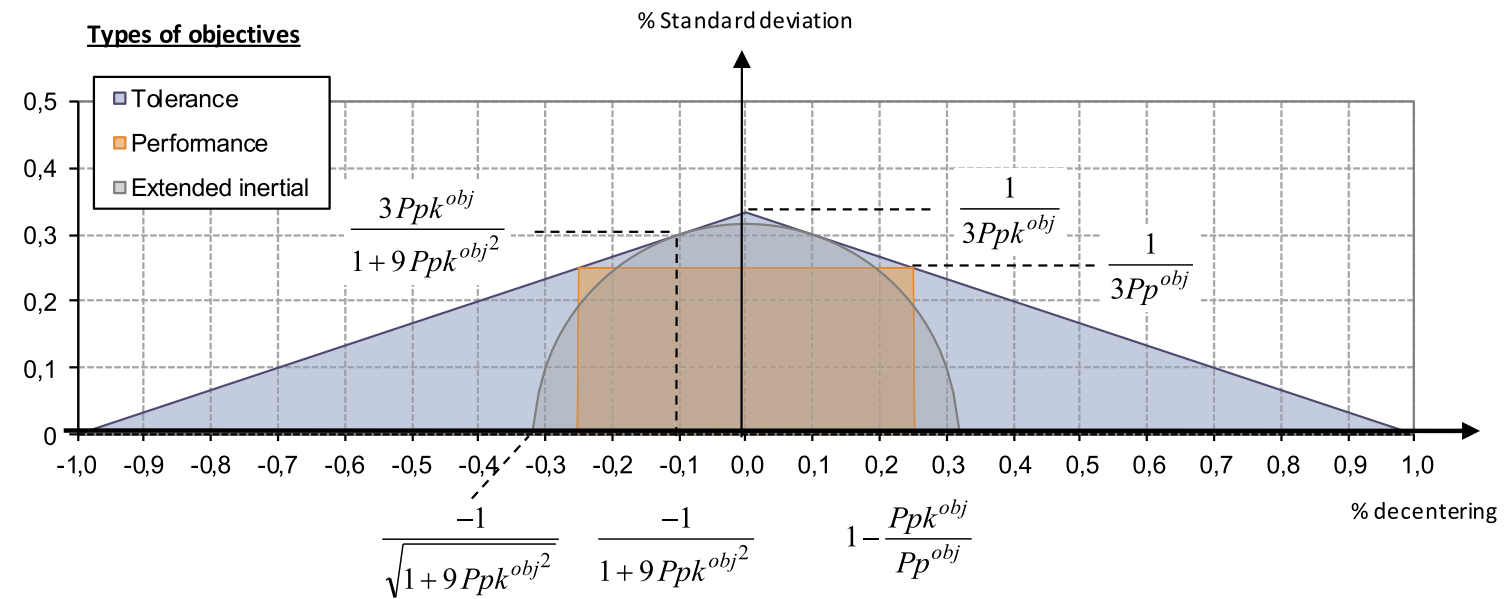

Fig. 3. Illustration of surfaces of maximum conformity.

$$
\begin{aligned}
P p_{Y} & =\frac{I T}{6 \sigma_{Y}}=\frac{R}{3 \sigma_{Y}}, P p k_{Y}=\frac{R-\left|\delta_{Y}\right|}{3 \sigma_{Y}}, \text { and } P p m_{Y} \\
& =\frac{R p_{Y}}{3 \sqrt{\sigma_{Y}^{2}+\delta_{Y}^{2}}}=\frac{{ }^{1+9\left(P p_{Y}-P p k_{Y}\right)^{2}}}{\sqrt{1+p^{2}}}
\end{aligned}
$$

For some real objectives $P p^{o b j} \geq P p k^{o b j}>0$ and $P p m^{o b j}=\sqrt{\frac{1}{9}+P p k^{o b j 2}},{ }^{10}$ the writing of the characteristic conformity $(Y, v, R)$, for each of the preceding conformations with maximum objectives, takes the following form (Fig. 3).

$$
\text { Tolerance - conformed at } P p k^{o b j} \text { if } P p k_{Y} \geq P p k^{o b j} \text {. }
$$

\footnotetext{
${ }^{10}$ These real numbers are chosen objectives and the reason for their ratings is due to the fact that the conformity criteria are expressed in a simplified way using the capability indexes. However, it should not be assumed that these objectives are capability indexes associated with objective decentralization concepts and objective standard deviation. These are numbers chosen to express the compliance criteria outlined.
}

Performance-conformed at $\left(P p^{o b j}, P p k^{o b j}\right)$ if

$$
\frac{P p k_{Y}}{P p k^{o b j}}>\frac{P p_{Y}}{P p^{o b j}} \geq 1 \text {. }
$$

Inertial - conformed at $P p m^{o b j}$ if $P p m_{Y} \geq P p m^{o b j}$.

The surface associated with an inertial objective is a semi-circle [7-9].

For usual objectives: $P p k^{o b j}=1, P p^{o b j}=\frac{4}{3} \approx 1,33$ and $\operatorname{Ppm}^{o b j}=\frac{\sqrt{10}}{3} \approx 1054$.

The usual conformity defined by inequalities $P p_{Y} \geq$ $P p^{o b j}$ and $P p k_{Y} \geq P p k^{o b j}$ and corresponding to the trapezoidal surface delimited by the triangle and the top of the rectangle at Figure 3 suffers from the same defect as the tolerance-conformity, by leaving too much latitude to the allowed decentring [10].

In the example (Fig. 3), the characteristic is extended inertial, tolerance-compliant and performance-compliant. Rectangle and Ellipse are inside the triangle and cover the available area.

The study of the advantages and disadvantages of conformities in terms of characteristic acceptability is not the subject of this paper [11]. However, by way of example, 
In the annexes (property 3), we describe a specific property of the conformity in inertial tolerancing (Inertial-conformed) that performance-conformed does not verify.

\section{Statistical tolerancing and conformity principles}

The overall problem is defined like this: for any $n$-uplets of charactertics $\left(Y_{i}\right)_{i=1 \ldots n}$ with the centered nominals independently, $Y_{J e u}$ to the centered nominal verifying (1) and $v_{\text {Clearance }}$ verifying $(3)$, what are the optimal $R_{\text {Jeu }}$ which ensure the principle of conformity?

When the same type of objective is used for $Y_{\text {Clearance }}$ and $Y_{i}$, the principle of conformity is said to be "under the assumption of judgment identity", which we will assume later. $^{11}$

The conformity principle in the worst case results in:

$$
\text { If for any } i=1 \ldots n,\left|Y_{i}-v_{i}\right| \leq R_{i} \text { p.s. }
$$

$$
\text { then }\left|Y_{\text {Clearance }}-v_{\text {Clearance }}\right| \leq R_{\text {Clearance }} \text { p.s. }
$$

As $\left|Y_{\text {Clearance }}-v_{\text {Clearance }}\right| \leq \sum_{i=1}^{n}\left|a_{i}\right|\left|Y_{i}-v_{i}\right|$, the preceding condition is verified when the arithmetic tolerancing formula is used.

On the other hand, there is no smaller value for $R_{\text {Clearance }}$ enabling this condition to be satisfied. ${ }^{12}$ Therefore, without taking any risks, if an assembly of worst-case parts conformed to the worst case, only the arithmetic tolerance is optimal. Another advantage of arithmetic tolerancing is that it is adapted to a set of conformities including those presented above, without necessarily being optimal.

Property 1-Conformity principle and arithmetic tolerancing.

For $C$ a conformity with concave objective type, if the characteristics $\left(Y_{i}\right)_{i=1 \ldots n}$ are independent and $C$-conformed, then $Y_{\text {Clearance }}$ is $C$-conformed for arithmetic tolerancing $R_{\text {Clearance }}=\sum_{i=1}^{n}\left|a_{i}\right| R_{i}{ }^{13}$

This result shows that the arithmetic tolerancing makes a separation between the design specification and the verification of these manufacturing specifications possible. In practice, the considered conformities always have concave types of objective. This concavity aims to

\footnotetext{
${ }^{11}$ Using the same types of objectives is not a theoretical but reasonably practical obligation. It is indeed difficult to manage distinct types according to the characteristics.

${ }^{12}$ If $Y_{i}$ are conformed at the worst case scenario, then $-R_{i} \leq \delta_{i}$ $\leq R_{i}$ for any $i=1 \ldots n$. then $\left|\delta_{\text {Clear }}\right| \leq \sum_{i=1}^{n}\left|a_{i}\right| R_{i}$, which requires $\sum_{i=1}^{n}\left|a_{i}\right| R_{i} \leq R_{\text {Clear }}$. Indeed, if not, with $\delta_{i}=\operatorname{sgn}\left(a_{i}\right) R_{i}$, we would have $\quad \delta_{\text {Clear }}=\sum_{i=1}^{n}\left|a_{i}\right| \delta_{i}=\sum_{i=1}^{n}\left|a_{i}\right| R_{i} \sum_{i=1}^{n}>R_{\text {Clear }}$, which would contradict the fact that $Y_{\text {Clear }}$ is conformed in the worst case.

13 This is an immediate check using inequality $\sqrt{\sum_{i=1}^{n} x_{i}^{2}} \leq \sum_{i=1}^{n} x_{i}$
for any $\left(x_{i}\right)_{i=1 \ldots n} \in \mathbb{R}^{n}$.
}

guarantee the second inequality of (6) on the functional requirement from this same inequality on each of the elementary characteristics. The demonstrations of this property are given in annexe 3 for better readability.

Arithmetic tolerancing suffers, however, from a functional incoherence that reflects its severity [12]: centered and fair characteristics that satisfy the conformity, or not, can induce conformal clearances for objectives well above what is required.

Is there a less stringent criterion of conformity than the worst case for statistical tolerance? The positive answer to this question, and which is subject to the following property (shown in the appendix), is a remarkable result. However, its application is difficult because the choice of a tolerancing formula and the choice of the criterion and the objectives of conformity to be used can no longer be decoupled.

Property 2-Conformity principle and statistical tolerance.

Are $\left.n \in \mathbb{N}^{*},\left(\left(c_{i}\right)_{i=1 \ldots n}, c_{\text {lear. }}\right) \in\right] 0,1\left[^{n+1} \quad\right.$ and, $C_{i}=\left(k_{i}\right.$, $\left.\left(c_{j} r, \xi_{i}^{o b j}\right)\right)$ for any $i=1 \ldots n$ and $C_{\text {Clear. }}=\left(k_{\text {Clear. }},\left(c_{\text {Clear. }} r\right.\right.$, $\left.\left.\xi_{\text {Clear. }}\right)\right)$ conformities such as $g: u \rightarrow r(\sqrt{u})^{2}$ concave.

Is $\left(\left(\lambda_{i}\right)_{i=1 \ldots n}, \lambda_{\text {Clear. }}\right) \in \mathbb{R}_{+}^{*}{ }^{n+1}$ checking the following constraints:

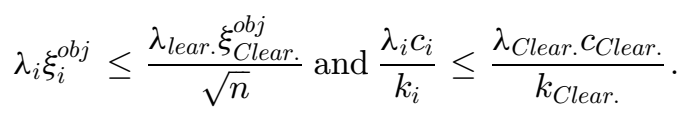

If the characteristics $\left(Y_{i}\right)_{i=1 \ldots n}$ are independent and $C_{i^{-}}$ conformed, then $Y_{\text {Clear. }}$ is $C_{\text {Clear.-conformed for: }}$

$$
R_{\text {Clear. }} \geq \lambda_{\text {Clear. }} \sqrt{\sum_{i=1}^{n} a_{i}^{2}\left(\frac{R_{i}}{\lambda_{i}}\right)^{2}} .
$$

This property means that, under a strong constraint on objectives, for any linear combination of $n$ independent characteristics, statistical tolerancing $(5)^{14}$ makes it possible to verify the principle of conformity for selected conformities. The assumption of concavity of $g: u \vec{r}(\sqrt{u})^{2}$ is verified for rectangular or elliptic but not triangular conformity. It is because the rectangular or elliptical conformities impose a control of the decentring that the situation is very different from that in which the decentring are only constrained by the limits of the TIs, which is the case for the tolerance-conformed, for example.

Let us illustrate this in the usual case where $P p^{o b j} \geq$ $P p k^{o b j}>0$ are the conformity objectives for the $Y_{i}$ et $P p_{\text {Clear. }}^{o b j} \geq P p k_{\text {Clear. }}^{o b j}>0$ those for $Y_{\text {Clear. }}$. If $Y_{\text {Clear. verifies }}$ (1) and $v_{\text {Clear. }}$ verifies (3), $\frac{P p_{\text {Clear. }}^{o b j}}{P p^{o b j}} \sqrt{\sum_{i=1}^{n} a_{i}^{2} R_{i}^{2}}$ is the minimum of $R_{\text {Clear } .} \in \mathbb{R}_{+}^{*}$ ensuring the following equivalence ${ }^{15}$ :

\footnotetext{
${ }^{14}$ For any $i=1 \ldots n, \lambda_{i}=1, \lambda_{\text {Clear }}=\frac{6}{h}$.

${ }^{15}$ This is a consequence of the situation where the offsets and standard deviations of $Y i$ are at the limits. Property ensures the reciprocity with the conformity of the maximum performance.
} 
Performance-Conformity principle (under the assumption of judgment identity):

$$
\begin{aligned}
& \text { If for any } i=1 \ldots n \frac{\left|\delta_{i}\right|}{R_{i}} \leq 1-\frac{P p k^{o b j}}{P p^{o b j}} \text { and } \frac{\sigma_{i}}{R_{i}} \leq \frac{1}{3 P p^{o b j}} \\
& \text { then } \begin{aligned}
\frac{\left|\delta_{\text {Clear. }}\right|}{R_{\text {Clear. }}} & \leq 1-\frac{P p k_{\text {Clear. }}^{o b j}}{P p_{\text {Clear. }}^{o b j}} \text { and } \frac{\sigma_{\text {Clear. }}}{R_{\text {Clear. }}} \\
& \leq \frac{1}{3 P p_{\text {Clear. }}^{o b j} .}
\end{aligned}
\end{aligned}
$$

\section{Constraint of realization:}

$$
P p^{o b j}-P p k^{o b j} \leq \frac{1}{\sqrt{n}}\left(P p_{C l e a r .}^{o b j}-P p k_{C l e a r .}^{o b j}\right) .
$$

By allowing intelligently controlled risks on all $Y_{i}$, statistical tolerancing can be used without impairing the quality of the functional requirement. However, the difficulty of exploiting this result is related to the dependence of the constraint of production on the number of characteristics $n$. It therefore requires setting objectives according to the least favourable situation for characteristics belonging to several linear relationships.

\section{Conclusions}

The problem in using statistical tolerancing lies in the control of the decentring of the characteristics at the time of their production. This is where using a statistical tolerance reaches its limits and is the only reason that obliges the designer to always preferentially examine the acceptability of a clearance with arithmetic tolerancing. For the latter, there is no taking of risk because if each characteristic is tolerant-compliant (lowest condition) then the clearance will necessarily be tolerant-compliant. This is no longer assured as soon as the tolerance of the functional requirement is calculated by a quadratic method.

However, statistical tolerancing could reduce the functional inconsistency of arithmetic tolerancing if, for example, performance-compliance was rigorously used with targets that were severe enough to maintain low decentring. This does not, however, prevent the use of a statistical tolerance without a mind-set: it allows the designer to widen the tolerances of the links for an acceptable clearance, which satisfies the manufacturer in terms of feasibility. Everyone seems satisfied, except that the question should be asked: who pays for the uncontrolled risks? Firstly, the company that manages the crises does so at great expense, but also the customer through chronic dissatisfaction that costs them very dearly. In other words, everyone! Suffice to say that the prospects offered by the regular reflections on industrial efficiency open the doors to an information management that remains to be agile.

We have shown in this paper that statistical tolerancing must be used in conjunction with a specific definition of conformity that we have defined. In addition, we have shown the importance of the concave objective types to satisfy the principle of conformity using statistical tolerancing.

\section{Appendices}

\section{Mathematical development}

Property 2 - Conformity principle and statistical tolerancing

Are $\left.n \in \mathbb{N}^{*},\left(\left(c_{i}\right)_{i=1 \ldots n}, c_{\text {lear. }}\right) \in\right] 0,1\left[^{n+1} \quad\right.$ and,$C_{i}=\left(k_{i}\right.$, $\left.\left(c_{i} r, \xi_{i}^{o b j}\right)\right)$ for any $i=1 \ldots n$ and $C_{\text {Clear. }}=\left(k_{\text {Clear. }}\right.$, $\left.\left(c_{\text {Clear. }}, \xi_{\text {Clear. }}\right)\right)$ conformities such as $g: u \rightarrow r(\sqrt{u})^{2}$ concave.

Is $\left(\left(\lambda_{i}\right)_{i=1 \ldots n}, \lambda_{\text {Clear. }}\right) \in \mathbb{R}_{+}^{* n+1}$ checking the following constraints:

$$
\lambda_{i} \xi_{i}^{o b j} \leq \frac{\lambda_{\text {Clear. }} \xi_{\text {Clear. }}^{o b j} \text { and }}{\sqrt{n}} \frac{\lambda_{i} c_{i}}{k_{i}} \leq \frac{\lambda_{\text {Clear. }} c_{\text {Clear. }} .}{k_{\text {Clear. }}} .
$$

If the characteristics $\left(Y_{i}\right)_{i=1 \ldots n}$ are independent and $C_{i^{-}}$ conformed, then $Y_{\text {Clear. }}$ is $C_{\text {Clear. }}$-conformed for:

$$
R_{\text {Clear. }} \geq \lambda_{\text {Clear. }} \sqrt{\sum_{i=1}^{n} a_{i}^{2}\left(\frac{R_{i}}{\lambda_{i}}\right)^{2}} .
$$

A - If for any $i=1 \ldots n,\left|\delta_{i}\right|<\xi_{i}^{o b j} R_{i}$ then $\left|\delta_{\text {Clear. }}\right|<\xi_{\text {Clear. }}^{o b j} R_{\text {Clear. }}$. This is a consequence of the first constraint and the inequality $\sqrt{\sum_{i=1}^{n} x_{i}^{2}} \leq \sum_{i=1}^{n} x_{i}$. Indeed:

$$
\begin{aligned}
\left|\delta_{\text {Clear. }}\right| & \leq \sum_{i=1}^{n}\left|a_{i}\right|\left|\delta_{i}\right|<\sqrt{n} \lambda_{\text {Clear. }} \xi_{\text {Clear. }}^{o b j}\left(\frac{1}{n} \sum_{i=1}^{n}\left|a_{i}\right| \frac{R_{i}}{\lambda_{i}}\right) \\
& \leq \sqrt{n} \lambda_{\text {Clear. }} \xi_{\text {Clear. }}^{o b j} \sqrt{\frac{1}{n} \sum_{i=1}^{n} a_{i}^{2}\left(\frac{R_{i}}{\lambda_{i}}\right)^{2}} .
\end{aligned}
$$

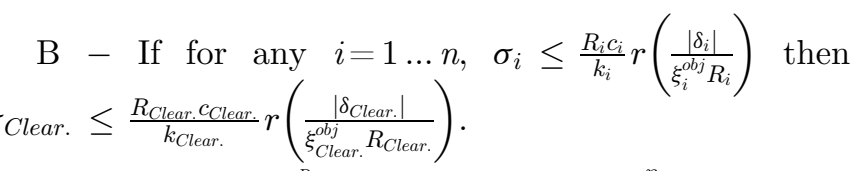

For $\beta_{i}=\frac{\left|a_{i}\right| \frac{R_{i}}{\lambda_{i}}}{\sqrt{\sum_{i=1}^{n} a_{i}^{2}\left(\frac{R_{i}}{\lambda_{i}}\right)^{2}}} \geq 0$, we have $\sum_{i=1}^{n} \beta_{i}^{2}=1$ and $\beta_{i} \geq \frac{\lambda_{\text {Clear. }}}{R_{\text {Clear. }} .}\left|a_{i}\right| \frac{R_{i}}{\lambda_{i}} \geq \frac{\sqrt{n} \xi_{i}^{\text {obj }}}{\xi_{\text {Jeu }}^{\text {obj }} R_{\text {Clear. }}}\left|a_{i}\right| R_{i}$.

Using the $g$ definition, the second constraint and the inequality on $R_{\text {Jeu }}$ :

$$
\begin{aligned}
& \sigma_{\text {Clear. }}^{2} \leq \sum_{i=1}^{n}\left(\frac{\lambda_{i} c_{i}}{k_{i}}\right)^{2} a_{i}^{2}\left(\frac{R_{i}}{\lambda_{i}}\right)^{2}\left(r\left(\frac{\left|\delta_{i}\right|}{\xi_{i}^{o b j} R_{i}}\right)\right)^{2} \\
& \leq\left(\frac{R_{\text {Clear. }} . c_{\text {Clear. }}}{k_{\text {Clear. }}}\right)^{2} \\
& \sum_{i=1}^{n} \frac{a_{i}^{2}\left(\frac{R_{i}}{\lambda_{i}}\right)}{\sum_{i=1}^{n} a_{i}^{2}\left(\frac{R_{i}}{\lambda_{i}}\right)^{2}}\left(\sum_{i=1}^{n}\left(\beta_{i} \frac{\left|\delta_{i}\right|}{\xi_{i}^{o b j} R_{i}}\right)^{2}\right) \\
& \leq\left(\frac{R_{\text {Clear. }} . c_{\text {Clear. }}}{k_{\text {Clear. }}}\right)^{2} g\left(\left(\frac{\left|\delta_{i}\right|}{\xi_{i}^{\text {obj }} R_{i}}\right)^{2}\right)
\end{aligned}
$$


By concavity of $g$ then using its decreasing and the inequality between arithmetic mean and quadratic

$$
\begin{aligned}
\left(\frac{1}{n} \sum_{i=1}^{n} x_{i}\right. & \left.\leq \sqrt{\frac{1}{n} \sum_{i=1}^{n} x_{i}^{2}}\right): \\
\sigma_{\text {Clear. }}^{2} & \leq\left(\frac{R_{\text {Clear. } c_{\text {Clear. }}}}{k_{\text {Clear. }}}\right)^{2} g\left(\sum_{i=1}^{n}\left(\beta_{i} \frac{\left|\delta_{i}\right|}{\xi_{i}^{o b j} R_{i}}\right)^{2}\right) \\
& \leq\left(\frac{R_{\text {Clear. }} c_{\text {Clear. }}}{k_{\text {Clear. }}}\right)^{2} g\left(\left(\frac{1}{\sqrt{n}} \sum_{i=1}^{n} \beta_{i} \frac{\left|\delta_{i}\right|}{\xi_{i}^{o b j} R_{i}}\right)^{2}\right) .
\end{aligned}
$$

Finally, by definition of $g$ and decay of:

$$
\begin{aligned}
\sigma_{\text {Clear }}^{2} & \leq\left(\frac{R_{\text {Clear. }} c_{\text {Clear }}}{k_{\text {Clear }}}\right)^{2} r^{2}\left(\frac{1}{\sqrt{n}} \sum_{i=1}^{n} \frac{\sqrt{n \xi_{i}^{o b j}}}{\xi_{\text {Jeu }}^{o b j} R_{\text {Jeu }}}\left|a_{i}\right| R_{i} \frac{\left|\delta_{i}\right|}{\xi_{i}^{o b j} R_{i}}\right)^{2} \\
& \leq\left(\frac{R_{\text {Clear. }} c_{\text {Clear }}}{k_{\text {Clear }}}\right)^{2} r^{2}\left(\frac{\left|\delta_{\text {Clear. }}\right|}{\xi_{\text {Clear. }}^{\text {obj }} R_{\text {Clear. }}}\right)
\end{aligned}
$$

Below, we present the coherence property of the inertial-conformity.

\section{Definition 2 - Characteristic conditionally conformed.}

For $(Y, v, R)$ a characteristic, a $Y$ 's process $E(Y / M)=$ $M(Y, M)$ admits a joint law, $E(Y / M)=M$ and $\frac{Y-M}{\sqrt{V(Y / M)}}$ is independent of $M$. The notation $Y / M$ will mean $Y$ conditionally to $M$ process of $Y$. if:

Is $C=\left(k,\left(r, \xi_{r}^{o b j}\right)\right)$ a conformity. $Y / M$ is $C$-conformed

$$
\begin{gathered}
M \in B\left(v, \xi_{r}^{o b j} R\right) p . s . \\
\text { and } \forall x \in B\left(v, \xi_{r}^{o b j} R\right) \sigma_{Y / M}(x) \leq \frac{R}{k} r\left(\frac{\left|\delta_{Y / M}(x)\right|}{\xi_{r}^{o b j} R}\right)
\end{gathered}
$$

with, for all real $x: \delta_{Y / M}(x)=E(Y / M=x)-v$ and $\sigma_{Y / M}(x)=\sqrt{V(Y / M=x)}$.

A conformity $C$ is said to be coherent if for any characteristic $(Y, v, R)$ and $M$ a $Y$ 's process, if $Y / M$ is $C$-conformed then $Y$ is $C$-conformed. It reflects the facts that the mixing of conforming production batches remains conformed.

The consistency of a conformity is not verified in general (if $Y / M$ is $C$-conformed, $\mu_{Y}=E(Y)=E(E$ $(Y / M))=E(M) \in B\left(v, \xi_{r}^{o b j} R\right) \quad\left(\right.$ since $M \in B\left(v, \xi_{r}^{o b j} R\right)$ p.s.) but $\sigma_{Y / M}\left(\mu_{Y}\right) \leq \frac{R}{k} r\left(\frac{\left|\delta_{Y / M}\left(\mu_{Y}\right)\right|}{\xi_{r}^{o b j} R}\right)$ does not generally impose $\sigma_{Y} \leq \frac{R}{k} r\left(\frac{\left|\delta_{Y}\right|}{\xi_{r} b j}\right)$.

The following lemma is used for the demonstration of the coherence property below.

\section{Lemma - The types of concave lenses.}

Is $r:[0,1] \rightarrow \mathbb{R}$ a continuous application. The following assertions are equivalent:
1. $r$ is decreasing concave.

2. For any v.a.r. $U \in[-1,1] p . s . E(r(|U|)) \leq r(E(U))$.

$1 \Rightarrow 2$ : By inequality of Jensen, ${ }^{16}$ as $r$ is concave, $E(r(|U|)) \leq r(E(|U|))$. Furthermore $r$ is decreasing and $|E(U)| \leq E(U)$.

$2 \Rightarrow 1$ : We pose $g=r \circ \|:[-1,1] \rightarrow \mathbb{R}$. For any continuous $f:[0,1] \rightarrow[-1,1]$ and uniform $U$ on $[0,1], f(U) \in[-1,1]$ $p . s$. and by assumption:

$$
\begin{aligned}
E(g \circ f(U)) & =\int_{0}^{1} g(f(t)) d t \leq g\left(\int_{0}^{1} f(t) d t\right) \\
& =g(E(f(U)) .
\end{aligned}
$$

Are $(a, b) \in{ }^{[-1,1] 2}, a<b, \lambda \in[0,1], \varepsilon \in[0, \min (\lambda, 1-\lambda)]$ and $f_{\varepsilon}:[0,1][a, b]$ defined by:

$f_{\varepsilon}$ is continuous and $\int_{0}^{1} f_{\varepsilon}(t) d t=\lambda b+(1-\lambda) a$. Further-
more: $\int_{0}^{1} g\left(f_{\varepsilon}(t)\right) d t=(\lambda-\varepsilon) g(b)+(1-\lambda-\varepsilon) g(a)+\int_{\lambda-\varepsilon}^{\lambda+\varepsilon} g\left(f_{\varepsilon}(t)\right) d t$.

But $g$ is continuous on the compact $[a, b]$ because $r$ is continuous, it thus reaches a minimum $m \in \mathbb{R}$ and $\int_{\lambda-\varepsilon}^{\lambda+\varepsilon} g\left(f_{\varepsilon}(t)\right) d t \geq 2 \varepsilon m$. Consequently, according to (19):

$$
\begin{aligned}
& \lambda g(b)+(1-\lambda) g(a)-2 \varepsilon\left(\frac{g(a)+g(b)}{2}-m\right) \\
& \quad \leq g(\lambda b+(1-\lambda) a) .
\end{aligned}
$$

This inequality being true for any $\varepsilon \in] 0, \min (\lambda, 1-\lambda)[$, we deduce, by definition, that $g=r \circ \|$ is concave on $[-1$, 1]. $r$ is therefore concave. Moreover it is decreasing on $[0,1]$ since for any $(\lambda, v) \in[0,1]^{2}$ :

$r(\nu)=1-\lambda \quad 2 g(-v)+\left(1-\frac{1-\lambda}{2}\right) g(v) \leq g\left(-\frac{1-\lambda}{2} \quad \nu+\right.$ $\left.\left(1-\frac{1-\lambda}{2}\right) \nu\right)=r(\lambda \nu)$

Note that if $r(0)>0$ and $r(1) \geq 0$, the concavity of $r$ ensures that it is strictly positive on $[0,1[$.

\section{Property 3 - Characterization of a conformity coherency.}

Are $k \in \mathbb{R}_{+}^{*}$ and $r:[0,1] \rightarrow[0,1]$ a continuous application such as $0<r(0) \leq \frac{k}{\sqrt{1+k^{2}}}$.

The following assertions are equivalent:

1. $C=\left(k,\left(r, \xi_{r}^{o b j}\right)\right)$ is a coherent conformity.

2 . Is exists continuous concave decreasing $f:[0,1] \rightarrow] 0$, 1] as $f(0)=1, \xi_{r}^{o b j}=\frac{\sqrt{f(1)} r(0)}{k} \leq 1$ and for any $u \in[0,1]$ $r(u)=r(0) \sqrt{f(u)-f(1) u^{2}}$.

$1 \Rightarrow 2$ : Are $U \in[-1,1]$ p.s., $v \in \mathbb{R}, R \in \mathbb{R}_{+}^{*}$. We pose:

$$
\begin{aligned}
M & =v+\xi_{r}^{o b j} R U \in B\left(v, \xi_{r}^{o b j} R\right) \text { p.s. and } \forall x \in B\left(v, \xi_{r}^{o b j} R\right) \sigma(x) \\
& =\frac{R}{k} r\left(\frac{|x-v|}{\xi_{r}^{o b j} R}\right) .
\end{aligned}
$$

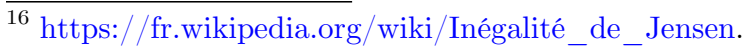


There is then a characteristic $(Y, v, R)$ of $M$ process Mas: $V(Y / M)=\sigma^{2}(M)$. By definition, $Y / M$ is $C$-conformed and then, by coherence of $C, Y$ is $C$-conformed, that is, noting that $\delta_{Y}^{2}+\sigma_{Y}^{2}=E\left(\delta_{Y / M}^{2}+\sigma_{Y / M}^{2}\right)$ :

$$
\begin{gathered}
E\left((M-v)^{2}+\frac{R^{2}}{k^{2}} r^{2}\left(\frac{|M-v|}{\xi_{r}^{o b j} R}\right)\right) \\
\leq\left(\mu_{Y}-v\right)^{2}+\frac{R^{2}}{k^{2}} r^{2}\left(\frac{\left|\mu_{Y}-v\right|}{\xi_{r}^{o b j} R}\right)
\end{gathered}
$$

Then, $\quad E\left(\left(\xi_{r}^{o b j} k U\right)^{2}+r^{2}(|U|)\right) \leq\left(\xi_{r}^{o b j} k E(U)\right)^{2}+r^{2}$ $(|E(U)|)$ and the lemma ensures that the application $g$ : $u \in[0,1] \rightarrow\left(\xi_{r}^{o b j} k u\right)^{2}+r^{2}(u) \in \mathbb{R}_{+}^{*}$ is concave decreasing.

Especially: $\left(\xi_{r}^{o b j} k\right)^{2}\left(1-u^{2}\right) \leq r^{2}(u) \leq r^{2}(0)$ because $r(1)=0$.

Also, there are $\lambda \in[0,1]$ as $\xi_{r}^{o b j}=\frac{\lambda r(0)}{k} \leq 1$ and $\left.\left.f: u \in[0,1] \frac{\overrightarrow{g(u)}}{r^{2}(0)} \in\right] 0,1\right]$ checks 2.

$2 \Rightarrow 1$ : As for any $u \in[0,1] f(1) u^{2} \leq f(1) \leq f(u) \leq f(0)$ the application $r$ verifies: $r(0) \sqrt{f(1)} \sqrt{1-u^{2}} \leq r(u)=$ $r(0) \sqrt{f(u)-f(1) u^{2}} \leq r(0) \sqrt{1-f(1) u^{2}}$.

$C=\left(k,\left(r, \xi_{r}^{o b j}\right)\right)$ is a conformity. Indeed, $r:[0,1] \rightarrow[0,1]$ is strictly positive on $\left[0,1\left[, r(1)=0, \xi_{r}^{o b j} \in \mathbb{R}_{+}^{*}, r\right.\right.$ is decreasing because $f$ is decreasing and for any $u \in[0,1] r(u)+\xi_{r}^{o b j}$ $u \leq r(0)\left(\sqrt{1-f(1) u^{2}}+\frac{\sqrt{f(1)}}{k} u\right) \leq 1$ because $r(0) \leq$ $\frac{k}{\sqrt{1+k^{2}}}$.

Finally, for a characteristic $(Y, v, R) M$ process as $Y / M$ is $C$-conformed, $E(M)=\mu_{Y} \in B\left(v, \xi_{r}^{o b j} R\right)$ and:

$$
\begin{aligned}
\delta_{Y}^{2}+\sigma_{Y}^{2} & =E\left(\delta_{Y / M}^{2}+\sigma_{Y / M}^{2}\right) \leq \frac{R^{2} r^{2}(0)}{k^{2}} E\left(f\left(\frac{|M-v|}{\xi_{r}^{o b j} R}\right)\right) \\
& \leq\left(\mu_{Y}-v\right)^{2}+\frac{R^{2}}{k^{2}} r^{2}\left(\frac{\left|\mu_{Y}-v\right|}{\xi_{r}^{o b j} R}\right) .
\end{aligned}
$$

(The first inequality is a consequence of $Y / M C$ conformed and the second is an application of the inequality of Jensen with $f$ concave decreasing).
Therefore, $Y$ is $C$-conformed and $C$ is coherent.

\section{References}

1. M. Forestier, Vers une solution d'ensemble du problème de tolérancement, Revue de Statistique Appliquée 13, 83-92 (1965)

2. A. Hassani et al, Méthodologie d'analyse et d'optimisation des tolérances dans un contexte de conception intégrée, Mech. Indust. 9, 381-395 (2008)

3. V. Srinivasan, M.A. O'Connor, F.W. Scholtz, Techniques for Composing a Class of Statistical Tolerance Zones, in Advanced Tolerancing Techniques, Ed. Zhang (WileyInterscience, ISBN, 1997), vd. 471145947, pp. 139-165

4. E. Kureková, Measurement process capability, trends and approaches, Meas. Sci. Rev. 1, 43-46 (2001)

5. D. Grau, Testing capability indices for manufacturing processes with asymmetric tolerance limits and measurement errors, Int. J. Metrol. Qual. Eng. 2, 61-73 (2011)

6. V.E. Kane, Process capability indices, J. Qual. Technol. 18, (1986)

7. B.S. Gildeh, S. Asghari, Inertial capability index based on fuzzy, Int. J. Metrol. Qual. Eng. 2, 45-49 (2011)

8. M. Pillet, Inertial tolerancing in the case of assembled products, in Recent advances in integrated design and manufacturing in mechanical engineering (Springer, Netherlands, 2003), pp. 85-94

9. M. Pillet, Inertial tolerancing, TQM Magazine 16, 202 (2004)

10. W.A. Taylor, Process Tolerancing: a solution to the dilemma of worst-case versus statistical tolerancing, in Fall Technical Conference (1995)

11. J.M. Judic, Process Tolerancing: A new statistical tolerancing method for industrial processes not daily adjustable in mass production. Proposal of an improvement to Wayne Taylor's method, in Proceedings of IDMME, Virtual Concept, Bordeaux, France (2010)

12. M. Pillet, Améliorer la productivité : Déploiement industriel du tolérancement inertiel (Eyrolles, Edition d'Organisation, 2010)

13. J. Gilson, A New Approach to Engineering Tolerances (Machinery Publishing Co., London, England, 1951)

Cite this article as: Laurent Leblond, Maurice Pillet, Conformity and statistical tolerancing, Int. J. Metrol. Qual. Eng. 9, 1 (2018) 